\title{
The Near-Linear Regime of Gravitational Waves in Numerical Relativity
}

\author{
Peter Anninos ${ }^{(1)}$, Joan Massó ${ }^{(1,2)}$, Edward Seidel ${ }^{(1,3)}$, Wai-Mo Suen ${ }^{(1,4,5)}$, and Malcolm \\ $\operatorname{Tobias}^{(4)}$ \\ (1) National Center for Supercomputing Applications, 605 E. Springfield Ave., Champaign, \\ Illinois 61820 \\ (2) Department of Physics, Universitat de les Illes Balears, Palma de Mallorca E-07071, Spain \\ (3) Department of Physics, University of Illinois at Urbana-Champaign \\ (4) McDonnell Center for the Space Sciences, Department of Physics, Washington University, St. \\ Louis, Missouri 63130 \\ (4) Physics Department, Chinese University of Hong Kong, Hong Kong
}

(August 25, 2018)

\begin{abstract}
We report on a systematic study of the dynamics of gravitational waves in full 3D numerical relativity. We find that there exists an interesting regime in the parameter space of the wave configurations: a near-linear regime in which the amplitude of the wave is low enough that one expects the geometric deviation from flat spacetime to be negligible, but nevertheless where nonlinearities can excite unstable modes of the Einstein evolution equations causing the metric functions to evolve out of control. The implications of this for numerical relativity are discussed.
\end{abstract}

04.25Dm, $04.30+\mathrm{x}$

Typeset using REVTEX 
Introduction. The study of gravitational waves and their interactions, i.e., the dynamics of spacetime in its pure vacuum form, is important for both theoretical and observational reasons. However, due to its mathematical complexity and the need for previously unavailable large scale computational resources, apart from a few specialized solutions, this area of research is largely unexplored. Previous analytic and numerical work on pure gravitational wave spacetimes, done in $1 \mathrm{D}$ or $2 \mathrm{D}$, has led to many interesting results [1,2]. These discoveries raise interesting questions about waves in 3D.

Gravitational waves are also about to open up a fundamentally new area of observation: gravitational wave astronomy. A new generation of detectors [3] should see waves for the first time near the turn of the century. Even though the observed waves are expected to be weak here on Earth, they will have been generated in regions with strong, highly dynamic and nonlinear gravitational fields. It is therefore essential to study accurately both the strong and weak field regimes, as well as the long term secular behavior in transitory intermediate regimes.

Thus we have been motivated to undertake a systematic study of gravitational waves in 3D numerical relativity over the past few years. 3D studies of general gravitational wave spacetimes are different from lower dimensional studies in two very important respects. First, unlike in lower dimensional studies, there are no assumed symmetries to fix the gauge. One has to consider the full set of gauge freedoms during the numerical evolution. Second, 3D calculations are bound to have lower resolution, and hence larger errors. This in turn makes it difficult to separate physical from numerical and gauge effects. These two effects are in fact related, as finite differencing errors tend to excite all gauge freedoms available. The excitation of unstable modes, whether they correspond to gauge freedoms or not, is the underlying principle of the phenomena discussed in this paper.

We have developed advanced 3D Cartesian numerical codes, using different numerical techniques as well as different formulations of the Einstein equations, to study gravitational waves. Full details of our methods and codes will be reported elsewhere [4]. In this paper we focus on one important lesson we learned with the large amount of numerical studies 
carried out using these codes.

When the amplitude of the gravitational waves is low, the evolution is linear. At high amplitudes there are nonlinear geometric effects: the energy-momentum carried by the waves themselves changes the geometry of the spacetime so that the evolution cannot be described by linear equations. We have seen both of these regimes in our studies, as expected. What was not anticipated is that beyond the linear regime, but before the amplitude is large enough for nonlinear geometric effects to be significant, there exists a regime where the evolution of waves, as described by the full nonlinear Einstein equations, can differ dramatically from the evolution described by the linearized versions of the Einstein equations. This is what we called the "Near-Linear Regime" (NLR). The aim of this paper is to draw attention to the existence of such a regime.

Many questions immediately come to mind: In what way do these effects show up, how do they arise, and can they be controlled? What are the boundaries between the linear, near-linear, and nonlinear regimes? What are the implications in the numerical study of gravitational waves, and gravitational systems in general? Will such effects be important for the numerical simulation of waves as weak as those we expect to observe astrophysically? These are the questions we address in this paper.

Statement of the problem. The evolution of the metric functions is described by the Einstein equations in the standard $3+1$ form

$$
\begin{aligned}
\partial_{t} g_{i j}= & -2 \alpha K_{i j}+\nabla_{i} \beta_{j}+\nabla_{j} \beta_{i} \\
\partial_{t} K_{i j}= & -\nabla_{i} \nabla_{j} \alpha+\alpha\left(R_{i j}+K K_{i j}-2 K_{i m} K_{j}^{m}\right) \\
& +\beta^{m} \nabla_{m} K_{i j}+K_{i m} \nabla_{j} \beta^{m}+K_{m j} \nabla_{i} \beta^{m} .
\end{aligned}
$$

The question is: When will this evolution be different from what is described by the linearized evolution equations, namely, by taking $g_{i j}=\delta_{i j}+\epsilon h_{i j}$, and keeping only terms to first order in $\epsilon$ in Eqs.(11)? More precisely, we ask when the discrete versions of Eqs. (11) yield results significantly different from the discrete versions of the linearized equations, using the same finite differencing techniques. We use the same initial data, which satisfies the initial 
constraint equations, for both the nonlinear and linear evolutions.

The purpose of this paper is to demonstrate, with concrete examples, that significant nonlinear effects appear before one might expect. Without careful analysis, these effects can be mistaken for true geometric effects, e.g, the formation of a spacetime singularity. These effects are due to unstable nonlinear modes admitted by Eqs. (1) (and/or their finite differenced versions), excitable by waves even with quite low amplitudes.

Quadrupole waves. The first example is of quadrupole waves similar to those studied in [5], except that we use an imploding-exploding wave configuration which is time symmetric [6], and we take the metric given in [5] as the conformal part of the metric function. We then determine the conformal factor using the York decomposition to solve the hamiltonian constraint equation [7]. The time symmetry of this imploding-exploding data automatically guarantees that the momentum constraint is also satisfied.

We study a case where the wave is initially centered at the origin, with a peak amplitude of $A \equiv g_{i j}-\delta_{i j} \sim 10^{-3}$, and a width of order unity (we use $c=G=1$ throughout this paper). The evolution of this initial data is predictable: As the amplitude of the wave is of the order $A \sim 10^{-3}$, and both space and time derivatives can bring in only factors of order unity, the energy density (e.g. Landau-Lifshitz pseudo energy density) must be of order $A^{2} \sim 10^{-6}$. With this "wave energy density" concentrated in a spatial dimension of order unity, the strength of the "gravitational potential M/R" (or spacetime curvature), can at most be of order $10^{-6}$. This is approximately $10^{6}$ times too weak to produce a significant effect on the propagation of the wave, e.g. to "bend" the null characteristics of the spacetime. Hence we expect the linear wave to expand outward just as in flat space. The scattering of the wave by itself, and any tail effects would affect the metric functions to at most of order $A^{3} \sim 10^{-9}$, which is beyond the level of accuracy in our simulations. Hence the wave train should propagate outward basically in a linear fashion, except for a slight delay in phase.

This picture is confirmed in Fig. Ta where we show the evolution of a typical metric function $g_{x x}$ (solid lines) along the $z$ axis from the initial time $t=0$ to $t=3$, with zero shift and unit lapse. This is to be compared to what is obtained with the same initial data, 
but evolved with the linearized evolution equations (dotted lines). At early times, the two waveforms are completely indistinguishable.

Next we look at the evolution at late times in Fig. 1 $1 \mathrm{~b}$ and zoom into the origin where we expect the spacetime to have returned to flat space. The nonlinear results (solid lines) are basically one everywhere except for a dip near the origin. This is clearly due to nonlinear effects, as there is absolutely no dipping in the linearized treatment (dotted lines). There are similar dips in $g_{y y}$ and $g_{z z}$, while the off diagonal metric functions develop a shear-like structure. These "residual" features will keep growing in time until the code crashes. This behavior is independent of grid resolution and has a characteristic growth rate that increases in proportion to $A^{2}$.

What causes this result? There are four possibilities: (i) nonlinear spacetime geometric effects which are not captured by the order of magnitude estimate given above; (ii) nonlinearity coupled with finite differencing errors; (iii) nonlinearity coupled with gauge effects; (iv) bugs in the nonlinear evolution code. In principle, any of these possibilities can cause this feature.

After much investigation [4] that includes numerous code tests, convergence studies, comparisons with linear evolutions, different numerical techniques, and even different formulations of the Einstein equations, we conclude that, in this case, it is (iii). The nonlinearity excites an unstable gauge mode of the evolution equations. A posteriori, the effect is simple to understand. The potential well created by the initial wave packet, although quite small in amplitude, sets the coordinate lines into motion, drifting towards each other. As the wave propagates outward, the potential well (spacetime curvature) reduces to zero, but the coordinate lines, once set into motion, will keep drifting towards the center. Hence over time, the distance between, say, constant $x$ coordinate lines decreases and a dip in $g_{x x}$ develops.

At what point does this effect appear (the lower boundary of the NLR)? This depends on the amplitude of the wave and the length of time it is evolved. We have observed dipping for waves with magnitude $A \sim 10^{-4}$ and spatial extension the same as in Fig. Ia, after it is evolved to about $t=10$. 
At what amplitude would nonlinear geometric effects become significant (the upper

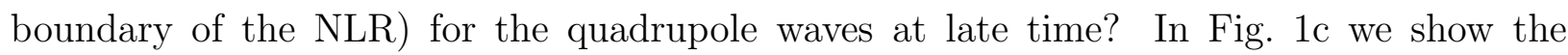
Riemann invariant $J$ [8] obtained using nonlinear and linear evolutions at time $t=4$, for various initial wave amplitudes $A$ 's. We see that despite the dipping of the metric, the invariants are basically the same up to $A \sim 0.05$. Beyond that, the linear and nonlinear results rapidly diverge, indicating nonlinear geometric effects.

The existence of unstable modes in the nonlinear evolution equations in the NLR highlights the importance of controlling the motion of the coordinates. In Fig. 11d we show the evolution of $g_{x x}$ at various times using the minimal distortion shift [9] and unit lapse. We see that there is no dipping. Details of this calculation and other methods in controlling the coordinate motion with both shift and lapse will be discussed elsewhere [4].

Colliding Wave Packets. Next we discuss the collision of two plane wave packets. In this case a completely different type of unstable mode is excited in the numerical evolution of Eqs. (1). The initial data is given by two nearly gaussian packets of the form shown by the solid line at $t=0$ in Fig. 2 for the metric function $g_{x x}$. The evolution is carried out with $\alpha=1$, zero shift, and grid spacing $\Delta x=0.05$. The wave packets, which are initially located at $z= \pm 3$, travel towards each other and collide at the center of the grid at $t=3$. In the wake of the waves, following the collision, we see that the metric function $g_{x x}$ develops an upward drift. Comparing $g_{x x}$ from the linear (dashed line) and nonlinear evolutions at $t=9$, we see clearly that the drift is a nonlinear effect.

Again we face the four possibilities pointed out above. In fact it is known that when two plane symmetric waves collide, a curvature singularity is generated due to the focusing effects of the waves [2] even for arbitrarily weak waves. However, it is easy to show, based on the colliding packet study in [2], that the singularity will develop at a time $t \sim \lambda^{2} /\left[(2 \pi A)^{2} \sigma\right]$ after the collision, where $\lambda$ is the characteristic wavelength, $\sigma$ is the characteristic width of the packet, and $A$ is the characteristic amplitude of the packet. For the case here, with $\lambda \sim 1, \sigma \sim 1$, and $A \sim 10^{-2}$, we expect the singularity to appear at $t \sim 250$, which is far beyond any evolutions shown here. Of course this estimate does not rule out that this drift 
is a "precursor" of the singularity, nor the possibility of other geometric nonlinear effects.

After much investigation [4] we confirm that the drift is an unstable mode in the nonlinear evolution equations. (而). We note that this is not in contradiction to the expectation that the Einstein equations are stable for weak waves (weak perturbations of the flat spacetime). It is the constraint equations that rule out these unstable modes. In our free evolution code the constraint equations are not enforced. This allows the unstable modes to develop after they are excited by the numerical errors in the evolution. The amount it is excited depends on the details of the numerical scheme. The instability causes the metric components in the wake of the waves to drift upwards according to $g_{i j}-\delta_{i j} \propto-\ln (C t+1)$. The time scale $1 / C$ of the instability depends strongly on the finite differencing scheme used. For the same run parameters used in Fig. 2, we find that $C=-1.6 \times 10^{-4}$ for a staggered leapfrog scheme, and a very different $C=7.9 \times 10^{-7}$ for a MacCormack scheme. We stress that the unstable mode exists on the level of the evolution equation and is excited in both of these two standard schemes, although with very different rates.

Conclusion. We have shown that there exists a "near-linear regime" (NLR) in the numerical study of waves in general relativity, in which the amplitude of the wave is weak enough so that nonlinear geometric effects are unimportant, but nevertheless unstable modes of the Einstein evolution equations can be excited by the nonlinearity. The instabilities manifest themselves as secular changes in the metric functions, which eventually grow to a level that affects the evolution, even crashing the code. There are various types of unstable modes. We have shown two examples of such modes [10]. In the quadrupole wave case, the instability is caused by nonlinearity coupled with gauge freedom. In the colliding plane wave packets case, the instability is caused by nonlinearity coupled with finite-differencing errors. The former effect conserves the constraint equations, the latter does not. In general, effects will be cross coupled and show up in many different forms. Further details of this analysis, as well as studies of other gravitational wave spacetimes will be given elsewhere. Our studies have been based solely on free evolutions. Unstable modes will be different for constrained evolutions. 
The aim of this paper is to draw attention to the existence of the NLR, so that the large amount of work leading to our keen awareness of these NLR phenomena need not be duplicated by other research groups in the field. We believe the instabilities in this regime are important in general for numerical relativity, with implications not just for gravitational waves, but also for evolving other 3D gravitational systems. The determining factor of whether nonlinear effects are important or not depends on both the strength of the gravitational potential and on the length of time the general relativistic system is evolved. To determine gravitational waveforms for astrophysical events, e.g., inspiral coalescences of compact binaries, which are expected to be one of the important observable sources of gravitational radiation, we would like to be able to evolve the system, compact objects and waves, to hundreds or even thousands of $M$, where $M$ is the mass of the system. For the success of such long time scale simulations, a full understanding and control of the kind of unstable modes pointed out in this paper are indispensable.

This research is supported by NCSA, PSC, and NSF grants Nos. PHY94-04788, PHY9407882, ASC95-03978 and ASC93-18152. 


\section{REFERENCES}

[1] K. Khan and R. Penrose, Nature 229, 185 (1971); F. Tipler, Phys. Rev. D 22, 2929 (1980); R. Matzner and F. Tipler, Phys. Rev. D 29, 1575 (1984); A. Abrahams and C. Evans, Phys. Rev. D 46, R4117 (1992); M. Choptuik, Phys. Rev. Lett. 70, 9 (1993).

[2] U. Yurtsever, Phys. Rev. D 37, 2790 (1988); Phys. Rev. D 38, 1731 (1988).

[3] A. A. Abramovici et al., Science 256, 325 (1992).

[4] P. Anninos, J. Massó, E. Seidel, W.-M. Suen and M. Tobias, Phys. Rev. D, in preparation.

[5] S. Teukolsky, Phys. Rev. D 26, 745 (1982).

[6] K. Eppley, in Sources of Gravitational Radiation, edited by L. Smarr (Cambridge University Press, Cambridge, England, 1979).

[7] J. York, in Sources of Gravitational Radiation, op cite.

[8] D. Kramer, H. Stephani, M. MacCallum and E. Herlt, Exact Solutions of Einstein's Field Equations (Cambridge University Press, Cambridge, England, 1980).

[9] L. Smarr and J. York, Phys. Rev. D, 17, 2529 (1978).

[10] We have just learned that similar instabilities with different wave configurations have also been observed by Shibata and Nakamura in their code (Phys. Rev. D 52, 5428 $(1995))$. 


\section{FIGURES}

FIG. 1. (a) The metric function $g_{x x}$ is plotted along the $z$ axis for a quadrupole wave. The wave peaks at the origin at $t=0$, and disperses outward. By $t=3$ the region near the origin returns basically to flat space, as expected. The same initial data is evolved with the nonlinear evolution equations (solid lines), and the linear evolution equations (dotted lines). (b) $g_{x x}$ is shown at later times $t=4,6,8,10$ for both linear (dotted lines) and nonlinear evolutions (solid lines). Note the development of a dip near the origin with the nonlinear evolution. (c) The Riemann invariant $J$ at the origin, is plotted against the initial amplitude of the wave $g_{z z}-1$, for both the nonlinear (solid line) and linear evolutions (dotted line) at $t=4$. At small amplitudes the two J's are the same, despite the dipping of the metric in the non-linear evolution. For perturbations of order $5 \%$ we start to see deviations, indicating the presence of nonlinear geometric effects. (d) An evolution of the same quadrupole data, but now evolved with the minimal distortion shift and $\alpha=1$. Note that the shift is able to hold the coordinate lines from drifting in.

FIG. 2. The metric function $g_{x x}$ is shown for colliding plane wave packets. The packets are initially centered at $z= \pm 3$ at $t=0$ and are moving towards each other. They collide at the origin at $t=3$, then pass through each other. The solid lines show the nonlinear evolution. Note the upward drift in the "wake" regions at $t=9$. Such drifting is absent in the linear evolution (dashed line). 


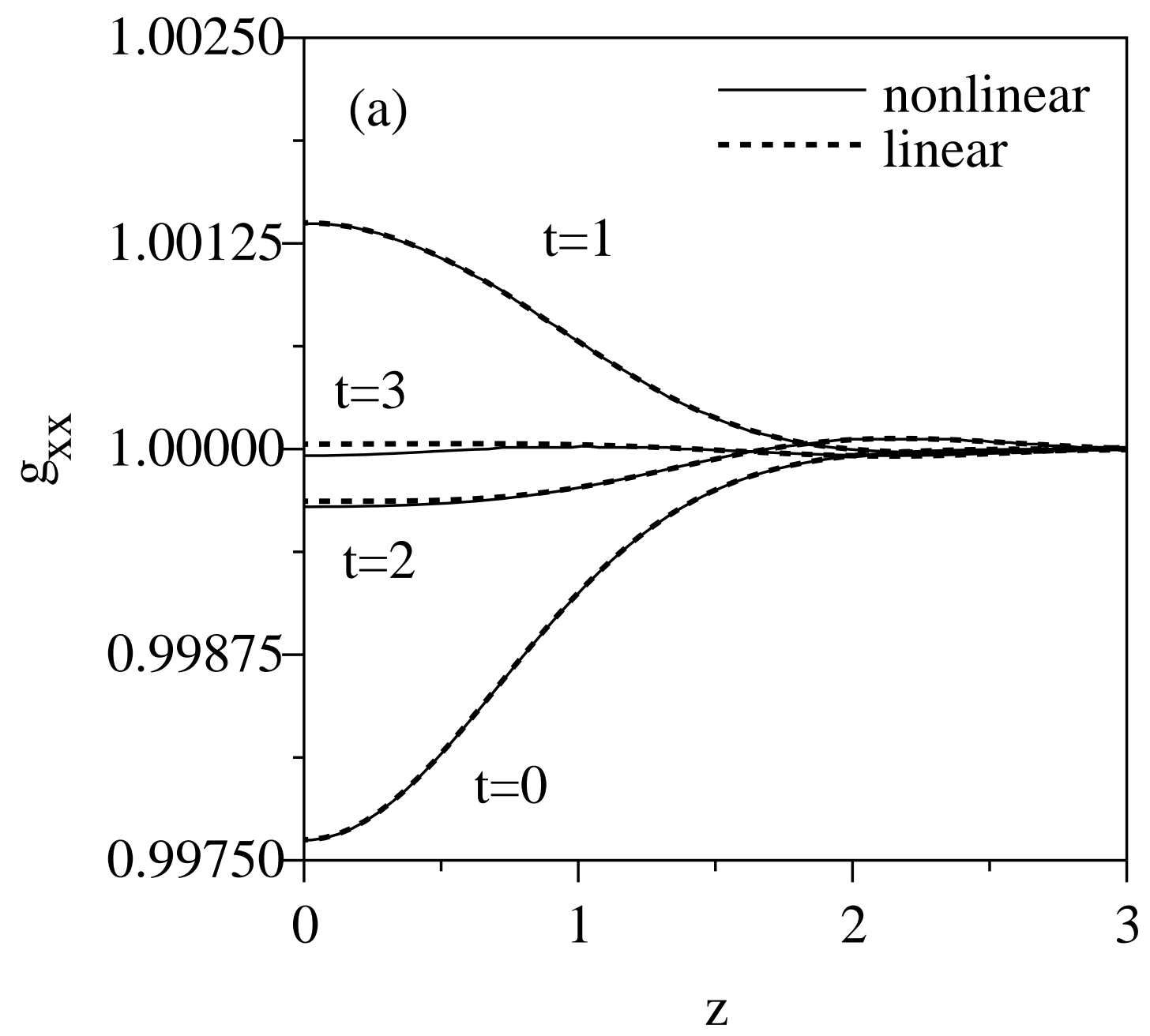




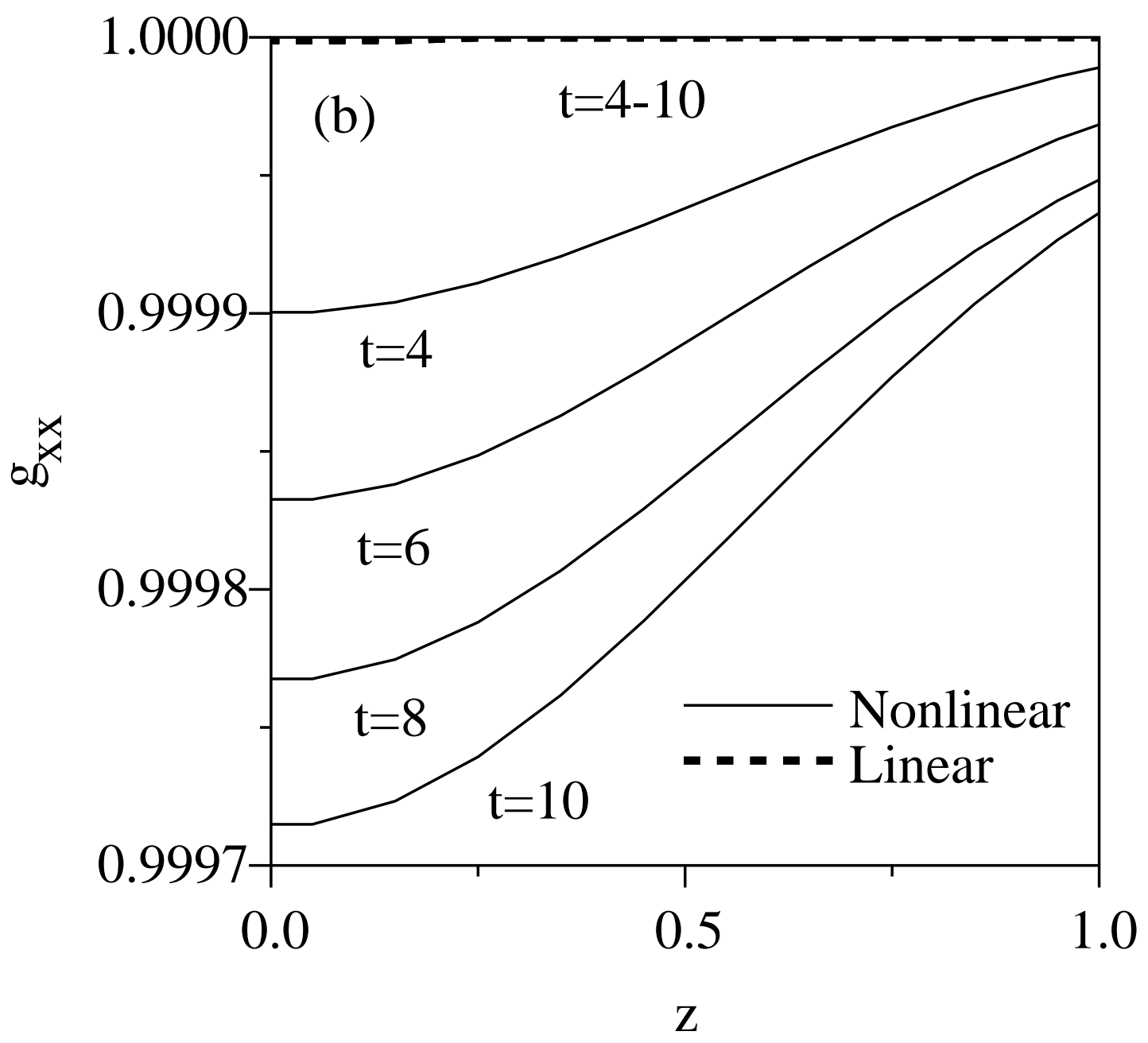




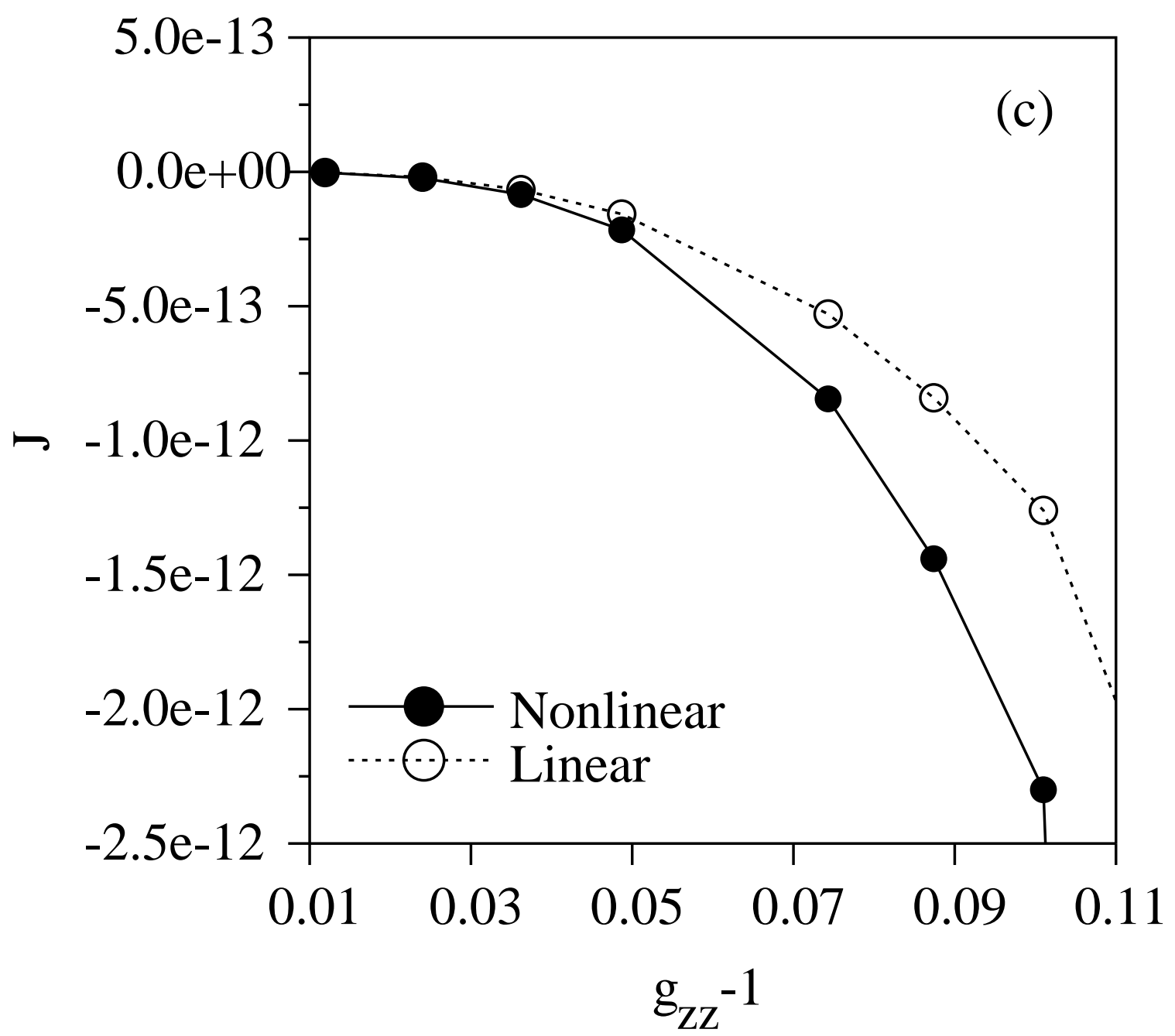




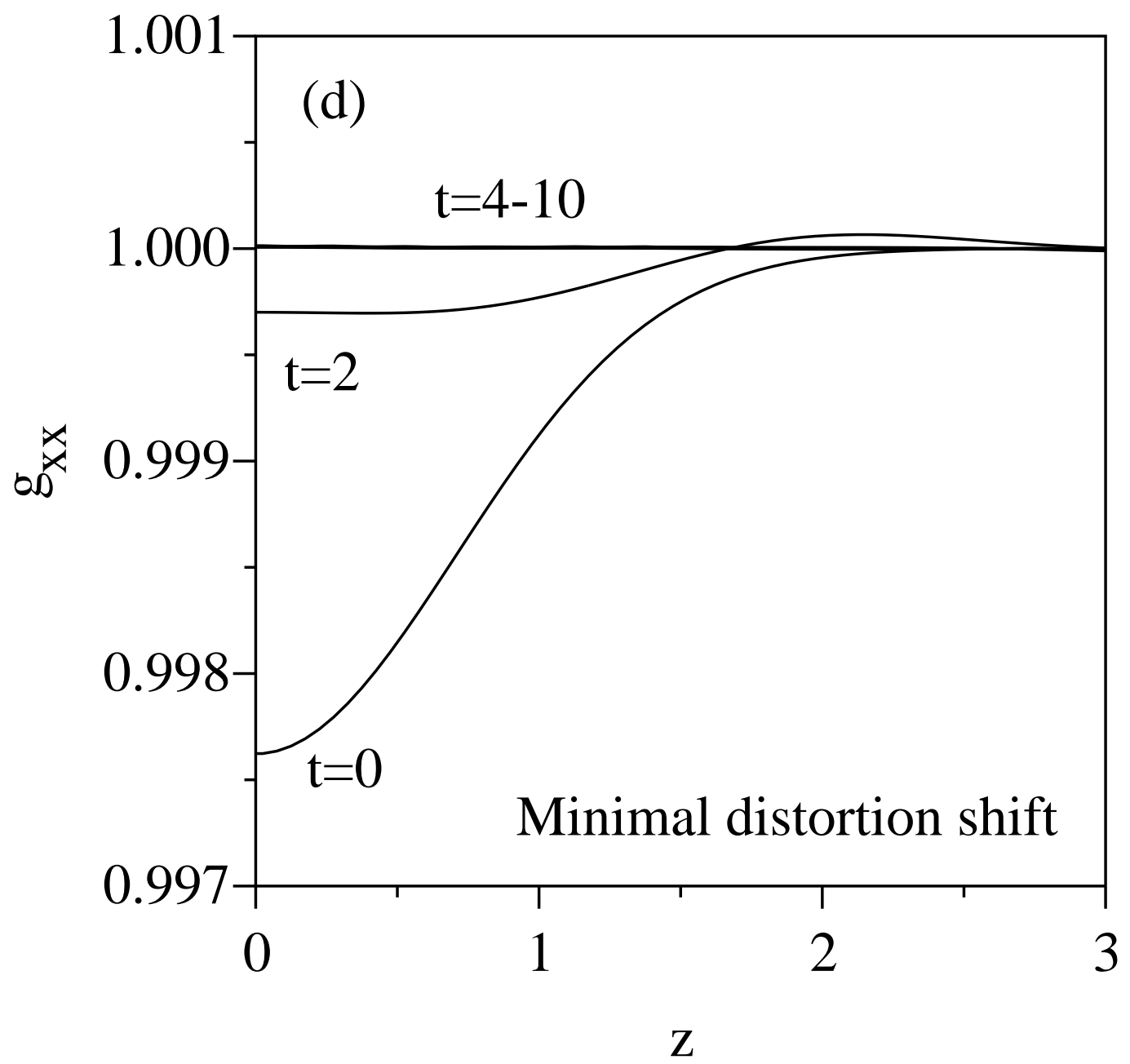




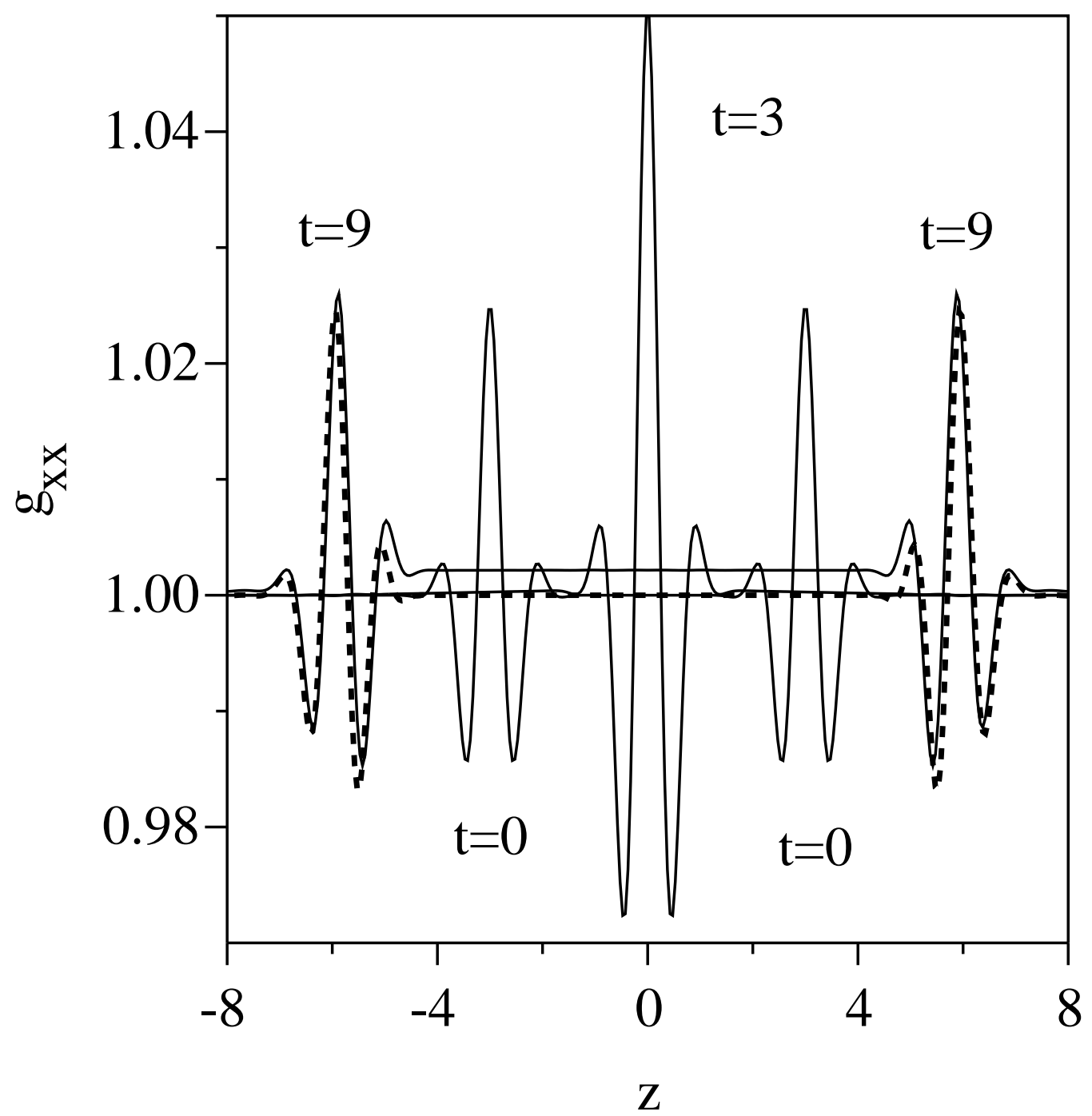

